\title{
De-programming colorectal cancer by increase in cholesterol level
}

\author{
Robert Skopec* \\ Researcher Analyst, AXON, Dubnik, Slovakia
}

\begin{abstract}
Complexities of cancers are representing another endless amount of new problems for the future development of novel treatments to prevent metastatic and secondary tumors. One of the biggest challenges in cancer treatment is the spread of cancer around the body, the metastasis. In many cases kills these secondary growths and not the original tumors. The main condition for secondary killers is given when cancer cells can break away from the primary place to travel around the organism and seed new tumors.
\end{abstract}

\section{Introduction}

Cholesterol and other fats are essential components of cell membranes which are playing a role in regulating processes such as cell migration and division. Cholesterol makes specialized membrane regions which are important regulators of cell function. Now the question is how these membrane regions increase cancer progression? It is statistically proved fact that the amount of cytoskeleton proteins at the cholesterol membranes is elevated in more of aggressive cancers in all models.

\section{Disentangling of metastasis from the tumor}

A key question in cancer research is how cancer cells are able to survive once they break away from a tumor and spread around the organism. As it can be well known, the cells are relatively protected when they are attached to other cancer cells and their surroundings. On the other hand, they become more vulnerable when they detach and float and normally undergo cell death.

Metastasis is currently incurable and remains one of the key targets of cancer research. Current research advances the knowledge of how two key molecules communicate and work together to help cancer cells survive during metastasis. This might lead also to the discovery of new drugs able to block the spread of cancer cells within the organism. This change could occur in cancer cells as they break away from tumors in cell cultures of zebrafish and mice. It was encountered a previously unknown survival mechanism in cancer cells that molecules known as integrins could be key.

\section{Integrins are proteins interacting with cells microenvironment}

Integrins are proteins on the cell surface that attach and interact with the cell's surroundings. Outside-in and inside-out signaling by integrins is known to help the cancer cells attach themselves surroundings. When the cancer cells are floating, like during the metastasis, the integrins switch. From their adhesion role to take on an entirely new form of communication: inside-in signaling, in which integrins signal within the cell.
An integrin $\beta 1$ (beta-1) pairs up with another protein c-Met and they move inside the cell together. The two proteins then travel to an unexpected location within the cell, normally used for a new role of cell communication. Then the two proteins send a message to the rest of the cell to resist against death and floating during metastasis. Using both breast and lung cells, metastases were less likely to form when $\beta 1$ and c-Met were blocked from entering the cell together, or were prevented from moving to the special location within the cell.

Integrins are already major targets for cancer treatment with drugs. Most integrin inhibitor drugs target their adhesive function and block them on the surface of the cancer cell. The limited success of these drugs could be partly explained by this newly discovered role of integrins within cancer cell.

A new strategy may prevent the integrin from going inside the cell the first place. These insights could lead to the design of better therapies against metastasis and more effective combinations that could prevent and slow both tumor growth and spread [1].

\section{Antioxidants feed the cancer}

Thought to protect cells from damaging by free radicals, antioxidants have long been touted to help prevent cancer. But outgoing from the new lab mice experiments indications, antioxidants actually accelerate the spread of skin cancer. The antioxidant foods were supposed to interact and neutralize free radicals, i. e. highly reactive chemicals that have the potential to harm cells. They were supposed to prevent cancer.

Today we must make some corrections in this line of causality effects, because if you have a cancerous cell, antioxidants may actually do more harm, than good, as the latest research is confirming this important difference.

Correspondence to: Robert Skopec, Researcher Analyst, AXON, Dubnik, Slovakia, E-mail: zxcbnvm7@gmail.com

Key words: colorectal cancer, cholesterol, tumor

Received: June 23, 2017; Accepted: July 13, 2017; Published: July 17, 2017 
Cancerous cells in lab mice with melanoma, the deadliest form of skin cancer, spread faster when they were administered antioxidants. During metastasis, an inefficient process, the vast majority of cancer cells fail to survive. That is changed in specialized mice with transplanted melanoma cells from patients, then fed $\mathrm{N}$-acetylcisteine (NAC), a man-made antioxidant used in nutritional and bodybuilding supplements. Administration of antioxidants to the mice allowed more of metastasizing melanoma cells to survive and increasing metastatic disease burden.

Unfortunately, most cancer patients die when their disease metastasizes. Prior studies have found that the metastasis of human melanoma cells in the specialized mice is predictive of their metastasis in human patients.

Antioxidants allow the body to prevent DNA damage from chemicals known free radicals, produced naturally by humans and found in leafy greens, vegetables and fruit. It appears antioxidants help cancer cells by fighting against a type of molecule in the human organism that can attack or damage then as they metastasize. Above research suggest that cancer patients should consider not supplementing their diets with the oxidation-fighting substances.

For humans with an increased risk of cancer, this means that taking nutritional supplements containing antioxidants may unintentionally speed up progression of small tumor or premalignant lesion, neither of which is possible to detect.

\section{Oxidative stress inhibits distant metastasis}

Solid cancer cells commonly enter the blood and disseminate systematically, but are highly inefficient at forming distant metastases. It was concluded in studies of human melanomas that differed in their metastasis histories in patients and in their capacity to metastasize NSG mice. Melanomas had high frequencies of cells that formed subcutaneous tumors, but much lower percentages of cells that formed tumors after intravenous or intrasplenic transplantation, particularly among inefficiently metastasizing melanomas.

Melanoma cells in the blood and visceral organs experienced oxidative stress not observed in established subcutaneous tumors.

Succesfully metastasizing melanomas underwent reversible metabolic changes during metastasis that increased their capacity to withstand oxidative stress, including increased dependence on $\mathrm{NADPH}$-generating enzymes in the folate pathway. Antioxidants promoted distant metastasis in NSG mice.

Folate pathway inhibition using low-dose methotrexate, ALDH1L2 knockdown, or MTHFD1 knockdown inhibited distant metastasis without specifically affecting the growth of subcutaneous tumors in the same mice. It is showing that oxidative stress limits distant metastasis by melanoma cells in vivo.

\section{Cholesterol link in aggressive cancers}

University of Queensland research has revealed that fats - including cholesterol could increase the progression of aggressive cancers. Their research showed a high cholesterol diet increased the spread of prostate cancer tumors to lymph nodes, lungs and bones, study leader M. Hill said. Cholesterol and other fats were essential components of cell membranes, and played a role in regulating processes such as cell migration and division.

Cholesterol makes specialized membrane regions-important regulators of cell function, and these membrane regions increase cancer progression. The amount of cytoskeleton proteins at the cholesterol membranes are elevated in more aggressive cancers in all models. The cytoskeleton is a network of fibres connecting parts of the cell, which plays an important role in structural integrity. Some chemotherapy drugs work on breaking up the cytoskeleton, i.e., the taxane family of drugs including docetaxel.

Because of its importance in all cells, targeting the cytoskeleton leads to toxicities and effects for patients. There is a need to identify a specific cancer-associated change in cytoskeleton, and then will be possible more specific therapies which can kill cancer cells without affecting normal cells. M. Hill's previous study on prostate cancer had revealed a reduction in the cytoskeleton-cholesterol membrane link when a gene that suppresses tumor growth was activated. When they were tested a completely unrelated tumor suppressor gene in an ovarian cancer cell model, they again found c correlation between reduction of cytoskeleton-cholesterol membrane linkage and cancer properties.

M. Hill had pointed out that above research reinforces the importance of cancer patients maintaining low cholesterol levels [2].

\section{Disentangling the association between statins, cholesterol, and colorectal cancer}

As it suggests a large case-control study from Penn Medicine researchers, long-term use of the cholesterol-lowering drugs statins does not appear to decrease a patient's risk of colorectal cancer. The observational analysis of over 100,000 patient's medical records suggests it is cholesterol levels that influence risk, not the muchdebated statins, that may explain the link between the widely-used cardiovascular drugs and risk.

Both statin use and high cholesterol have been linked to a lower colorectal cancer risk. Blood cholesterol levels were inversely related to colorectal cancer risk: the higher the cholesterol level, the lower the risk for patients, regardless of statin use, the authors find. They also found that an unexplained drop in cholesterol levels one year before a cancer diagnosis increased the risk of cancer in both statin users and nonusers. This unexplained drop in cholesterol may be caused by distant coincidences of the non-linear deviation term (NDT) directed by the Quantum Entanglement Entropy (QEE) effects [3-5].

The findings point a bigger role of cholesterol levels on cancer risk, it could serve as a blood biomarker to help diagnosis colorectal cancer earlier. Statins are a common cholesterol-lowering treatment strategy for the management of patients at risk for coronary heart disease. They did not account for the blood cholesterol level on cancer risk. They confirmed findings from previous studies showed a decreased risk of colorectal cancer in statin users compared to non-users. The difference in the risk of colorectal cancer was not significantly different between those patients who continued statin therapy and those who discontinued. For every $1 \mathrm{mmol} / \mathrm{L}(\sim 38.6 \mathrm{mg} / \mathrm{dl})$ increase in total cholesterol level, authors observed a 10 percent decreased risk of colorectal cancer.

They observed that decreases in total serum cholesterol $(>1$ $\mathrm{mmol} / \mathrm{L}$ ) at least a year before the cancer diagnoses were associated 1.25 -fold and 2.36-fold increased risk of colorectal cancer in users and nonusers respectively. These data demonstrate a complex association between statins, cholesterol, and colorectal cancer, caused by the QEE effects! While unexplained decreases in blood total cholesterol may be considered as connected to QEE, future studies are needed to determine 
the utility of blood cholesterol as a marker for early detection of cancer [6].

\section{The twofaced new main law of nature}

The quantum entanglement is a basis of twofaced reality in which we are living our lives. From this reality are outgoing also the science and healthcare too.Altoughmetastasis is important for systemic correlations expansion (as in tumors), it is a highly dichotomous process, with millions of cells being required to disseminate to allow for the selection of cells-correlates aggressive enough to survive the metastatic cascade. To quantify the dynamics of metastasis of correlations development, we need look at the incidence of metastases in terms of co-occurrence at every point of time. To quantify co-occurrence we can use the $\varphi$ -correlation between dichotomous variables defined as:

$$
\frac{N_{X}(t) C_{i j}(t)-m_{i}(t) m_{j}(t)}{\sqrt{m_{i}(t) m_{j}(t)\left[N_{X}(t)-m_{i}(t)\right]\left[N_{X}(t)-m_{j}(t)\right]}} \text { where } C_{i j}(t) \text { is the }
$$

number of co-occurrence at time t. Than $i$ and $j$ represent particular site of metastasis, $\mathrm{X}$ represents the primary correlations type. The pairwise correlations between metastasis network links for every primary correlations types and lead to the correlation coefficient matrix.

The dichotomous correlations of the adaptation may be caused also by the Quantum Entanglement Relative Entropy as a measure of distinguishability between two quantum states in the same Hilbert space. The relative entropy of two density matrices $p_{0}$ and $p_{1}$ is defined as $S\left(p_{1} \mid p_{0}\right)=\operatorname{tr}\left(p_{1} \log p_{1}\right)-\operatorname{tr}\left(p_{1} \log p_{0}\right)$. When $p_{0}$ and $p_{1}$ are reduced density matrices on a spatial domain $D$ for two states of a quantum field theory (QFT), implies that $S\left(p_{1} \mid p_{0}\right)$ increases with the size of $D$. Than $\Delta S_{E E}=-\operatorname{tr}\left(p_{1} \log p_{1}\right)+\operatorname{tr}\left(p_{0} \log p_{0}\right)$ is the change in entanglement entropy across $D$ as one goes between the states.

When the states umder comparison are close, the positivity is saturated to leading order: $S\left(p_{1} \mid p_{0}\right)=\Delta\left\langle H_{\text {mod }}\right\rangle-\Delta S_{E E}=0$.

The problem of conventional adaptation may be given by a definition of static, deterministic world. The proliferative correlations lead to the resonances between the degrees of freedom. When we increase the value of energy, we increase the regions where randomness prevails. For some critical value of energy, chaos appears: over time we observe the exponential divergence of neighboring trajectories. For fully developed chaos, the cloud of points generated by a trajectory leads to diffusion [7]. Here we must as first formulate a new Main Natural Law: the Quantum Entanglement Entropy (QEE) [3]. Through above resonances the QEE is causing a metastasis of correlations, antagonistically intertwining all types of potentially conflicting interests $[4,5]$.

\section{Red onions have a cancer-fighting effect}

University of Guelph researchers as first have discovered that Ontario-grown red onions have the strongest cancer-fighting power. They have tested five onion types grown in Ontario and discovered the Ruby Ring onion variety came out on top. Onions as a superfood are still not well known. But they contain one of the highest concentrations of quercetin, a type of flavonoid.

The Guelph study revealed that the red onion not only has high levels of quercetin, but also high amounts of anthocyanin, which enriches the scavenging properties of quercetin molecules. Anthocyanin is instrumental in providing colour to fruits and vegetables including the red onions which have the darkest colour, and have the most cancerfighting power. Published recently in Food Research International, the study involved placing colon cancer cellsin direct contact with extracted from the different onion varieties.

Study had proved that onions are excellent at killing cancer cells. Onions activate pathways that encourage cancer cells to undergo cell death. They promote an unfavorable environment for cancer cells and they disrupt communication between cancer cells, which inhibits growth. These findings follow a recent study by the researchers on new extraction technique that eliminates the use of chemicals, making the quercetin found in onions more suitable for consumption.

Developing a chemical-free extraction method is important because it makes possible to use onion's cancer-fighting properties in nutraceuticals and in pill form. This superfood is possible include in salads and on burgers as a preventive measure, onion extract will be added to food products such as juice or baked goods and be sold in pill form as a type of natural cancer treatment! $[8,9]$.

\section{Conclusions}

One of the many reasons tumors are so difficult to treat is that they are able to adapt whenever they are exposed to unfavorable conditions. Hypoxia as a lack of oxygen means a phenomenon that should weaken the tumor, but instead, the malignant cells are able to compensate and drive more aggressive disease behavior. Researchers at The Wistar Institute have identified a novel mechanism that selectively operates in hypoxic tumors to enable tumor cells to thrive and continue to proliferate despite a low oxygen environment. Hypoxia is a nearly universal hallmark of aggressive tumor growth.

Clinical trials in which cancer patients were administered antioxidants had to be stopped because the patients getting antioxidants were dying faster. The reason was: cancer cells benefit more from antioxidants than normal cells done.

The negative effects of antioxidants in cancer patients were pointed out in several similar studies. For example, water laced with NAC was linked to a doubling or three-fold increase in the number of lymphnode tumors in the mice who drank it, the team in Sweden found it. The same research team found that certain antioxidants can spur lung tumor growth in mice.

Other studies have linked high doses of antioxidant beta-carotene and form of vitamin A retinol, a 28-percent increased risk of lung cancer in smokers. High doses of vitamin E, another natural antioxidant, may increase risk of prostate cancer, according to the National Institute of Health.

Mitochondria, the "powerhouse" of cells because of their role in energy production, are the main source of hypoxia-induced reprogramming in tumors. As the Wistar lab showed that the protein Akt, which plays a key role in cell signaling and metabolism, accumulates in mitochondria during hypoxia. When it happens, the protein PDK1 is phosphorylated at a unique site, and a complex responsible for cellular respiration is shut down. The pathway then uses the tumor's metabolism to break down glucose and use its energy to reduce cell death and maintain proliferation. There are drugs that exist that specifically target Akt in cancer, and can impair a tumor's ability to adapt to hypoxia. Another practical pathway to impair tumors can be voluntarily reducing the consumption of glucose!

We must prove also the effects of Quantum Entanglement Entropy on biology of the cancer. It is possible due to inverselyrelated, paradoxical quantum effects such as the relation of the blood cholesterol to colorectal cancer risk, or another example of the more developed the diabetes leading to longer life of the patients, while if they are reducing 
more the consumption of the glucose, it can be shortening the life perspectives of the diabetes patients, etc.

Quantum physics says that the observer affects reality. Therefore, the patients can also change the way of observing reality that they are living in. Just posing the question, it changes the chemistry inside their body, as it pointed out Dr. F. Wolf. Changing the field of possibilities changes the pattern of behavior. With this approach of glucose reduction plus eating more red onions you can de-programming tumors.

David Chalmers, a philosopher at New York University regularly questions the reality that conscious minds perceive. The problem is that any evidence we could ever get would be also simulated...James Gates Jr. pointed out that such a simulation would mean reincarnation was possible-the simulation could always be run again bringing everybody back to life!It starts to break down a very funny barrier between what people often think is the conflict between science and faith.Philosopher Daniel Denett coined the term "the Cartesian Theater", what is a metaphor for the view of dualism of the QEE, originated by Descartes (inventor of the Cartesian coordinate system).

So we think that coded by "God's Code" means a selection, much more complex than Darwinian one, through the coding by Quantum Entanglement Entropy, lead to consciousness explained without oversimulation of the Cartesian Theater.

\section{Acknowledgments}

The author gratefully acknowledge the assistance of Dr. Marta Ballova, Ing. Konrad Balla, Livuska Ballova and Ing. Jozef Balla.

\section{Conflict of interest}

The author has no biomedical financial interest or a conflict of interest to declare related to this project. The paper is not supported by any grants.

\section{References}

1. Barrow-McGee R, Kishi N, Joffre C, Ménard L, Hervieu A, et al. (2016) Beta-1integrin-c-Met cooperation reveals an inside-in survival signaling on autophagy-related endomembranes. Nature Communications 7.

2. Shah AD, Inder KL, Shah AK, Cristino AS, McKie AB, et al. (2016) Integrative analysis of subcellular quantitave proteomics studies reveals functional cytoskeleton membrane-lipid raft interactions in cancer. J Proteome Res 15: 3451-3462.[Crossref]

3. Skopec R (2015) Intelligent Evolution, Complexity and Self-Organization. Neuro Quantology 13 .

4. Skopec R (2016) Fighting cancer with growing complexity. J Transl Sci 2

5. Skopec R (2017) Coding by Quantum Entanglement Entropy. Neuro Quantology 15.

6. Mamtani R, Lewis JD, Scott F, Ahmad T, Goldberg DS, et al. (2016) Disentangling the Association between Statins, Cholesterol, and Colorectal Cancer: A Nested CaseControl Study. PLoS Med 13: e1002007.[Crossref]

7. Prigogine I (1997) The End of Certainty. Time, Chaos, and the New Laws of Nature The Free Press, New York.

8. Petrie MC, Jhund PS, She L, Adlbrecht C, Doenst T, et al. (2016) Ten-Year Outcome After Coronary Artery Bypass Grafting According to Age in Patients with Heart Failure and Left Ventricular Systolic Dysfunction: An Analysis of the Extended Follow-Up of the STICH Trial (Surgical Treatment for Ischemic Heart Failure). Circulation 134: 1314-1324.[Crossref]

9. Doran CF, Faux MG, Gates SJ Jr, Hubsch T, Iga KM, et al. (2008) Relating DoublyEven Error-Correcting Codes, Graphs, and Irreducible Representations of N-Extended Supersymmetry. arXiv:0806.0051v1

Copyright: $(02017$ Skopec R. This is an open-access article distributed under the terms of the Creative Commons Attribution License, which permits unrestricted use, distribution, and reproduction in any medium, provided the original author and source are credited. 